\title{
Pancreatic islet isolation variables in non-human primates (rhesus macaques)
}

\author{
P. Andrades • C. K. Asiedu • B. Gansuvd • S. Inusah • \\ K. J. Goodwin • L. A. Deckard • U. Jargal • \\ J. M. Thomas
}

Received: 6 November 2007 / Accepted: 20 March 2008 / Published online: 9 May 2008

(C) Springer-Verlag 2008

\begin{abstract}
Background Non-human primates (NHPs) are important preclinical models for pancreatic islet transplantation (PIT) because of their close phylogenetic and immunological relationship with humans. However, low availability of NHP tissue, long learning curves and prohibitive expenses constrain the consistency of isolated NHP islets for PIT studies. To advance preclinical studies, we attempted to identify key variables that consistently influence the quantity and quality of NHP islets.

Methods Seventy-two consecutive pancreatic islet isolations from rhesus macaques were reviewed retrospectively. A scaled down, semi-automated islet isolation method was used, and monkeys with streptozotocin-induced diabetes, weighing 3-7 kg, served as recipients for allotransplantation. We analysed the effects of 22 independent variables grouped as donor factors, surgical factors and isolation technique factors. Islet yields, success of isolation and transplantation results were used as quantitative and qualitative outcomes. Results In the multivariate analysis, variables that significantly affected islet yield were the type of monkey, pancreas
\end{abstract}

P. Andrades · C. K. Asiedu • B. Gansuvd - K. J. Goodwin •

L. A. Deckard $\cdot$ U. Jargal $\cdot$ J. M. Thomas

Division of Transplant Immunology, Department of Surgery,

University of Alabama at Birmingham,

Birmingham, AL, USA

S. Inusah

School of Public Health,

University of Alabama at Birmingham,

Birmingham, AL, USA

P. Andrades ( $\square)$

VH L107,

1670 University Boulevard,

Birmingham, AL 35294-0019, USA

e-mail: patoandrades@gmail.com preservation, enzyme lot and volume of enzyme delivered. The variables associated with successful isolation were the enzyme lot and volume delivered. The transplant result was correlated with pancreas preservation, enzyme lot, endotoxin levels and COBE collection method.

Conclusions Islet quantity and quality are highly variable between isolations. The data reviewed suggest that future NHP isolations should use bilayer preservation, infuse more than $80 \mathrm{ml}$ of Liberase into the pancreas, collect non-fractioned tissue from the COBE, and strictly monitor for infection.

Keywords Islet isolation - Islet transplantation .

Non-human primates
Abbreviations
IEQ islet equivalent
NHP non-human primate
PIT pancreatic islet transplantation
UW University of Wisconsin solution

\section{Introduction}

Pancreatic islet transplantation (PIT) can be effective in the treatment of type 1 diabetes in humans [1,2]. However, major obstacles to be overcome before PIT becomes a routine clinical therapeutic method include the need for multiple donors, various transplantation procedures and chronic immunosuppression. Consequently, strategies to solve these problems must be developed first in the preclinical setting [3]. Non-human primates (NHPs) are an important preclinical animal model because of their close phylogenetic and immunological relationship with humans. That is why many centres perform preclinical PIT in NHPs [3-7], but consistent 
isolation of pancreatic islets from NHPs remains difficult. The difficulties relate to low availability of primate tissue, a long learning curve, high costs and limited knowledge of parameters that have a major impact on the results of NHP islet isolation. These considerations have delayed large-scale NHP studies [8].

Many factors affect the quantity and quality of isolated pancreatic islets. Among them, donor characteristics, organ procurement, pancreas condition, and digestion and purification procedures are the most important variables influencing islet yield, viability and functionality [9]. To improve the efficacy of islet transplantation, further examination and enhancement of current islet isolation methods are essential. The identification of additional important factors will help modify protocols, improve transplantation results and speed up the transition into clinical practice. The purpose of this study was to review our experience with PIT in NHPs and assess the variables that determine a successful outcome.

\section{Methods}

Animals Between 1999 and 2007 we performed 72 pancreatic islet isolations from rhesus macaques of Chinese $(n=21)$ and Indian $(n=51)$ origin (Covance Research Products, Alice, TX, USA). The average donor age was $5.4 \pm 1.9$ years and body weight $6.9 \pm 2.6 \mathrm{~kg}$. A total of 18 donor monkeys had a positive past medical history because they had been included in previous studies with different drug protocols or surgical procedures. Rhesus macaques weighing 3-7 kg served as recipients. Diabetes was induced with a single high dose $(110-140 \mathrm{mg} / \mathrm{kg})$ of streptozotocin administered i.v. The dose used was $\leq 1,200 \mathrm{mg} / \mathrm{m}^{2}$, which has been shown to induce stable diabetes safely in rhesus macaques [10]. All recipient-donor combinations underwent prospective molecular typing for MHC class I and II alleles and were selected to have multiple MHC mismatches as described previously [7]. Experiments were conducted under the standards set by the National Institutes of Health (USA) and were approved by the local Institutional Animal Care and Use Committee.

Organ procurement Briefly, the splenocolic and splenorenal ligaments were divided and the tail of the pancreas was completely mobilised from the major curvature of the stomach. After liberating the duodenum and pancreatic head from the retroperitoneum, we identified, ligated and transected the bile duct, the gastroduodenal junction and the proximal jejunum. The pancreatic area was packed with ice and approximately $750 \mathrm{ml}$ of cold, filtered University of Wisconsin solution (UW) was infused through the abdominal aorta while the cava vein was transected for exsanguination. This reduced warm ischaemia time to almost zero. The pancreas was then separated from the duodenum, spleen and surrounding tissues. The pancreatic duct was cannulated with a 24-gauge catheter, and accessory ducts were identified and cannulated or ligated depending on their diameter. Intraoperative findings or events that could affect the procurement were recorded. The harvested pancreas was preserved with cold UW $(n=64)$ or the two-layer technique [11] $(n=8)$. Rapid transfer to the laboratory for processing ensured an average cold ischaemia time of $25-30 \mathrm{~min}$. The pancreas was weighed and assessed before Liberase infusion and placement in the digestion chamber. Pancreas quality was determined by subjective evaluation of its macroscopic characteristics (oedema, presence of fat, haemorrhages and burns).

Islet isolation Donor islets were prepared by the semiautomated Ricordi technique [12] with minor modifications as previously described [7]. Reconstituted Liberase HI $(0.47 \mathrm{mg} / \mathrm{ml})$ (Roche, Indianapolis, IN, USA) with $(n=44)$ or without $(n=28)$ DNAase $(0.6 \mathrm{mg} / \mathrm{ml}$ DNAse I; Sigma, St Louis, MO, USA), was injected into the pancreas through the cannulated duct (ductal infusion). Liberase solution was infused with a peristaltic pump set at $44 \mathrm{ml} / \mathrm{min}$ in 17 cases or manually in 53 cases. Enzyme was injected directly into poorly distended areas (interstitial infusion). The pancreas was placed into the digestion chamber filled with enzyme and connected to a circuit and pump for recirculation. Digestion was performed at $30-35^{\circ} \mathrm{C}$ with gentle agitation. The extent of digestion was tracked by regular sampling; digestion was stopped by adding $150 \mathrm{ml}$ of cold heatinactivated fetal bovine serum (FBS; Sigma Aldrich, St Louis, MO, USA) when three $150 \mu \mathrm{m}$ dithiazone-stained acinar-free islets were observed in the sample. Average digestion time was $15.5 \pm 5.45 \mathrm{~min}$. The digestate was diluted with cold RPMI 1640 (750 ml; Mediatech, Herdon, VA, USA), HEPES solution $1 \mathrm{~mol} / \mathrm{l}(25 \mathrm{ml}$; Sigma Aldrich) and FBS solution $(250 \mathrm{ml})$, with $(n=29)$ or without $(n=26)$ Pefabloc $(4 \mathrm{mmol} / 1$; Roche, Indianapolis, IN, USA), and centrifuged at $8^{\circ} \mathrm{C}$, $1,000 \mathrm{rpm}$ for $5 \mathrm{~min}$. After three washes, all pellets were combined and put in Eurocollins (Mediatech, Indianapolis, IN, USA) containing $20 \%$ FBS. The digestate was purified in a COBE blood processor (COBE Laboratories, Lakewood, CO, USA) on a discontinuous Euroficoll gradient (Mediatech, Indianapolis, IN, USA): $100 \mathrm{ml}$ at a density of $1.037,125 \mathrm{ml}$ at a density of 1.096 , and $250 \mathrm{ml}$ at a density of 1.108 . Effluent was collected from the COBE in three fractions $(n=19)$ or more than three fractions $(n=53)$. After two washes in cold CRML-1060 (Sigma Aldrich), FBS (100 ml/l), ciprofloxacin $(1.0 \mu \mathrm{g} / \mathrm{ml})$, insulin-transferrin-selenium (10 ml/1; Mediatech, Herdon, VA, USA), vitamin E $(10 \mu \mathrm{mol} / \mathrm{l})$ and $\mathrm{ZnSO}_{4}(16.7 \mu \mathrm{mol} / \mathrm{l})$ were added. The isolated islets were cultured in Miami Medium \#1A $(900 \mathrm{ml}$; Mediatech, Indianapolis, IN, USA), FBS $(100 \mathrm{ml})$, ciprofloxacin $(1.0 \mu \mathrm{g} / \mathrm{ml})$ and reduced glutathione $(10 \mathrm{mg} / \mathrm{l})$ at $37^{\circ} \mathrm{C}$ and $95 \% \mathrm{O}_{2}$. 
Islet characterisation Islet preparations were evaluated for yield, size, morphology, viability and purity on the day of isolation before transplantation. Isolated islets were counted as described previously [13], adjusted to $\sim 500$ islet equivalents (IEQs) normalised to $150 \mu \mathrm{m}$ diameter (IEQ150) per millilitre, and by the IEQ/g of pancreas weight before digestion. The residual undigested pancreas weight was not used for this calculation. Islet viability was examined by ethidium bromide/acridine orange staining and expressed as the ratio between green (living) cells and orange (dead) cells in each islet examined [14]. Purity was determined as the ratio between islets (stained with dithiazone) and exocrine tissue (unstained by dithiazone) [14]. Routine static incubation for insulin release by glucose stimulation was not used [14], nor was in vivo transplantation of NHP islets into chemically diabetic immunodeficient mice [4].

Isolations were classified as successful $(n=43)$ or nonsuccessful $(n=29)$. A successful isolation was defined as any isolation that met the following criteria: (1) islet yield $>8000 \mathrm{IEQ} / \mathrm{g}$ of pancreas; (2) adequate islet morphology evaluated independently by two observers. Three $200 \mu \mathrm{l}$ samples of the islet suspension were taken after purification and examined with an inverted phase-contrast microscope with a calibrated grid in the eyepiece (the isolation outcome evaluated the shape, border, integrity, stain and diameter of the islets, giving an overall morphology rating of good or bad [11]; thus, no graded scale was used); (3) viability and purity both $>90 \%$ [14]; and (4) sterility (negative Gram stain and conventional $24 \mathrm{~h}$ culture) or endotoxin level $<1.0 \mathrm{EU} / \mathrm{ml}$ (QCL-1000 Chromogenic LAL Endpoint Assay; Cambrex, Charles City, IO, USA) [15, 16].

We combined islet yield and islet quality into one broad definition, and in this way we were able to measure both elements of the isolation outcome. Measuring islet quality is not easy and is very subjective, but it is used frequently in the islet isolation literature with wide acceptance of its advantages and disadvantages. In our experience these criteria differentiated the results of the isolations and helped in the transplantation decision for the islets.

Pancreatic islet transplantation A total of 48 pancreatic islet allotransplants were completed. One donor was used for one recipient in 25 cases, one donor was used for two recipients in 11 cases, and two donors were used for one recipient in one case. At laparotomy, islet infusion was performed by gravity flow through a no. 8 French catheter placed in the portal vein [7]. A mean of $22.754 \pm 8.401 \mathrm{IEQ} / \mathrm{kg}$ per recipient was infused. No significant haemodynamic or portal vein pressure changes were observed. Transplantation was considered successful when primary non-function did not occur. Graft function was measured using blood glucose levels and insulin requirements. Only the first 2 weeks after transplantation were considered for this analysis because of differences in the immunosuppressive protocols used. A transplant was considered to have full function when blood glucose levels were below $11.1 \mathrm{mmol} / \mathrm{l}$ and no exogenous insulin was needed. Islet allografts were considered to have partial function when blood glucose levels were maintained with more than $50 \%$ reduction of preoperative exogenous insulin requirements. Acute rejection was considered to have occurred when normal postoperative function was achieved but there was a slow return to preoperative blood glucose levels and exogenous insulin requirements.

Statistical analysis Outcomes that were examined in this study were: (1) islet yield (IEQ); (2) successful versus nonsuccessful isolation; and (3) transplantation outcome. The variables analysed for association with these outcomes were: (1) donor variables (age, sex, weight, medical history, type of monkey); (2) procurement variables (surgery findings, pancreas weight, pancreas quality, pancreas preservation); and (3) isolation variables (enzyme lot, pancreas infusion method, volume delivered, ductal or interstitial infusion, time and temperature of exposure to the enzyme, use of DNAase or Pefabloc, COBE delivery). A complete definition of each variable is given in Table 1 .

The mean and standard deviation were used to describe continuous variables and frequencies were used to summarise categorical variables. First, a univariate analysis using ANOVA was done to determine which variables affected islet yield and transplantation outcomes. Multiple regression was then performed to explore the effects of all the variables on islet yield and obtain a regression equation that described the prediction of the criterion variable. Next, multiple logistic regression was used to evaluate the prognostic abilities of the variables with respect to isolation success and transplantation outcome, and to estimate the OR with the $95 \%$ confidence interval (CI). The OR compared the relative odds of the outcome condition of the exposed and non-exposed groups, and the $95 \% \mathrm{CI}$ is the range or variance where $95 \%$ of the OR results may be. An OR with a $95 \%$ CI below or above 1 was considered to be a protective or favouring factor. Comparison between successful and non-successful isolation groups was performed using the Mann-Whitney and Fisher tests for continuous and categorical variables, respectively. All comparisons were two-tailed and $p \leq 0.05$ was considered statistically significant. All calculations were performed using SPSS for Windows, Version 15.0 (SPSS, Chicago, IL, USA).

\section{Results}

Islet yield The number of isolated islets varied greatly between different isolations $(78,663 \pm 42,570$ IEQ; 8,920 \pm 
Table 1 Description of the variables studied

\begin{tabular}{|c|c|}
\hline Variable & Description \\
\hline \multicolumn{2}{|l|}{ Donor } \\
\hline Age & Age of donor at the time of isolation (years) \\
\hline Sex & Donor sex (male or female) \\
\hline Donor weight & Weight of donor at the time of isolation $(\mathrm{kg})$ \\
\hline Past medical history & $\begin{array}{l}\text { Donor comorbidities that may influence isolation. Examples: previous surgeries or medications (positive } \\
\text { or negative) }\end{array}$ \\
\hline Type of monkey & Monkey origin (Indian or Chinese) \\
\hline \multicolumn{2}{|l|}{ Procurement } \\
\hline Surgery findings & $\begin{array}{l}\text { Important deleterious events that occurred during pancreas harvest. Examples: multiple adhesions, } \\
\text { intraoperative bleeding, pancreatic lesion (positive or negative) }\end{array}$ \\
\hline Pancreas weight & Weight $(\mathrm{g})$ of pancreas immediately after harvest and cleaning of the organ \\
\hline Pancreas quality & $\begin{array}{l}\text { Subjective evaluation of pancreas macroscopic characteristics before isolation. A blind evaluator considered } \\
\text { oedema, presence of fat, haemorrhage, burns and overall pancreas aspect (adequate and inadequate). } \\
\text { No special scoring system was used }\end{array}$ \\
\hline \multicolumn{2}{|r|}{ 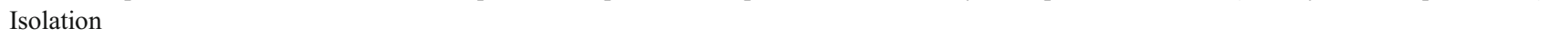 } \\
\hline Enzyme lot & $\begin{array}{l}\text { Lot number of the enzyme used during digestion. Lots were ordered chronologically and efficiency was } \\
\text { evaluated by determining the numbers }(\%) \text { of lots that allowed successful and non-successful isolation }\end{array}$ \\
\hline Infusion method & Method used for infusing the enzyme into the pancreas (by hand or using a pump) \\
\hline Volume delivered & Total volume $(\mathrm{ml})$ of enzyme infused into the pancreas \\
\hline Ductal infusion & Average percentage of enzyme volume infused into the pancreas through the pancreatic duct for digestion \\
\hline Interstitial infusion & Average percentage of enzyme volume infused into the pancreas interstitially for digestion \\
\hline Temperature & Mid-range temperature $\left({ }^{\circ} \mathrm{C}\right)$ recorded during the process of digestion in the Ricordi chamber \\
\hline Digestion time & Time (min) during which the pancreas was exposed to the enzyme in the digestion chamber \\
\hline DNAase & Additive to the solutions used during the isolation process (used and not used) \\
\hline Pefabloc & Additive to the solutions used during the isolation process (used and not used) \\
\hline COBE delivery & Method of collecting the samples after COBE purification $(<3$ or $>3$ fractions $)$ \\
\hline Endotoxin UW & Level of endotoxin in the UW transport solution of the pancreas after harvest $(\mathrm{EU} / \mathrm{ml})$ \\
\hline Endotoxin digest & Level of endotoxin in the digest $(\mathrm{EU} / \mathrm{ml})$ \\
\hline Culture time & Days of islet culture before transplant \\
\hline
\end{tabular}

5,062 IEQ/g pancreas). In the univariate analysis, the type of monkey, pancreas quality, pancreas preservation, enzyme lot, pump infusion method, volume delivered, use of DNAase and collection of three or fewer fractions after COBE delivery were significantly associated with superior islet yield (Table 2). Univariate analysis revealed no significant relationship between endotoxin levels in UW or Liberase and islet yield. Although there was wide variation in pancreas weight, this variable was not significantly associated with islet yield in the univariate or multivariate analysis, and similar results were observed when the analysis was performed using IEQ/g of pancreas instead of IEQ alone. In the multivariate analysis, the type of monkey $(p=0.0178)$, pancreas preservation $(p=0.0484)$, enzyme lot $(p=0.0056)$ and volume delivered $(p=0.0490)$ showed a statistically significant contribution to the variability of islet yield. Pancreases from Chinese monkeys yielded about 28,870 IEQs more than those from Indian monkeys. Two-layer pancreas preservation after procurement produced 33,845 IEQs more than UW preservation. Each millilitre of extra volume delivered into the pancreas increased islet yield by 116 IEQs. Islet yield decreased by 4,880 IEQs for each newer enzyme vial. Thus, there was a significant negative correlation between enzyme lot and islet yield (Spearman $r=-0.2551, p=0.033$ ). Analysis of the enzyme information provided by the manufacturer also showed a significant negative correlation between the enzyme lot and collagenase concentration (Spearman $r=-0.3822, p=0.003$ ) and a positive correlation between enzyme lot and endotoxin levels (Spearman $r=0.7646, p<0.001$ ). Therefore, islet yield diminished as the collagenase activity in the enzyme preparation decreased and endotoxin content increased.

Successful isolation As defined in the Methods, the successful islet isolation group showed higher islet yield, viability, purity and sterility than the non-successful islet isolation group (Table 3). Also, the successful isolation group resulted in a higher rate of transplantation. A total of $36(84 \%)$ successful isolations were used for transplantation and only seven were not transplanted because of their use in 
Table 2 Univariate analysis between isolation variables and islet yield and transplantation outcome

\begin{tabular}{|c|c|c|c|c|}
\hline Variable & Number of monkeys & Characteristic & Islet yield & Transplant outcome \\
\hline \multicolumn{5}{|l|}{ Donor variables } \\
\hline Age (years) & 72 & $5.4 \pm 1.9(2-14)$ & 0.454 & 0.735 \\
\hline Sex & 72 & Female, $6(8 \%)$; male, $66(92 \%)$ & 0.255 & 0.729 \\
\hline Donor weight (kg) & 65 & $6.9 \pm 2.6$ & 0.508 & 0.057 \\
\hline Medical history & 72 & Positive, 18 (25\%); negative, 54 (75\%) & 0.755 & 0.530 \\
\hline Type of monkey & 72 & Chinese, 21 (29\%); Indian, 51 (71\%) & $0.047 *$ & 0.491 \\
\hline \multicolumn{5}{|l|}{ Procurement variables } \\
\hline Surgery findings & 72 & Positive, 11 (15\%); negative, 61 (85\%) & 0.257 & 0.328 \\
\hline Pancreas weight $(\mathrm{g})$ & 72 & $9.5 \pm 3.5(1.8-19.6)$ & 0.059 & 0.923 \\
\hline Pancreas quality & 72 & Adequate, $36(50 \%)$; inadequate, $35(50 \%)$ & $0.001^{*}$ & 0.283 \\
\hline Pancreas preservation & 72 & UW, 64 (89\%); two-layer, 8 (11\%) & $0.023 *$ & $0.042 *$ \\
\hline \multicolumn{5}{|l|}{ Isolation variables } \\
\hline Enzyme lot & 70 & 20 chronologically ordered lots were used & $0.011 *$ & $0.042 *$ \\
\hline Infusion method & 72 & Hand, 53 (76\%); pump, $17(24 \%)$ & $0.025^{*}$ & 0.855 \\
\hline Volume delivered (ml) & 62 & $177.1 \pm 123.6(50-380)$ & $0.005^{*}$ & 0.486 \\
\hline Ductal infusion $(\%)$ & 65 & $80.6 \%$ & 0.650 & 0.135 \\
\hline Interstitial infusion (\%) & 65 & $19.4 \%$ & 0.775 & 0.147 \\
\hline Mean temperature $\left({ }^{\circ} \mathrm{C}\right)$ & 66 & $33.6 \pm 1.2(31.5-35.2)$ & 0.645 & 0.507 \\
\hline Digestion time (min) & 68 & $15.5 \pm 5.4(5-26)$ & 0.324 & 0.949 \\
\hline DNAase & 72 & No, 28 (39\%); yes, $44(61 \%)$ & $0.044^{*}$ & 0.296 \\
\hline Pefabloc & 55 & No, $26(47 \%)$; yes, $29(53 \%)$ & 0.499 & 0.831 \\
\hline COBE delivery & 72 & $<3$ fractions, $53(74 \%) ;>3$ fractions, $19(26 \%)$ & $0.000^{*}$ & 0.053 \\
\hline Endotoxin UW (EU/ml) & 46 & $0.3 \pm 0.3(0.01-1.5)$ & 0.698 & 0.051 \\
\hline Endotoxin digest $(\mathrm{EU} / \mathrm{ml})$ & 46 & $0.6 \pm 0.003(0.01-1.5)$ & 0.373 & $0.000 *$ \\
\hline Culture time (days) & 43 & 0 day, $5 ; 1$ day, $30 ; 2$ days, 8 & NA & 0.356 \\
\hline Viability $(\%)$ & 62 & $93.8 \pm 0.8$ & 0.221 & 0.370 \\
\hline Purity $(\%)$ & 62 & $94.7 \pm 0.6$ & 0.207 & 0.513 \\
\hline
\end{tabular}

NA, not applicable

$* p<0.05$

parallel studies. Only 2 of 29 (7\%) non-successful isolations were combined and transplanted into one recipient. There were no differences in donor and procurement variables between the groups. Among isolation variables analysed, only volume delivered, infusion method and enzyme lot showed statistical differences. More than $80 \mathrm{ml}$ of volume delivered into the pancreas was predictive of a successful isolation (OR 5.216, 95\% CI 1.746-15.586, $p=0.004$ ). There was no association between enzyme volume delivered and pancreas quality (Spearman $r=-0.04326, p=$ 0.736). The use of pump infusion showed borderline significance as a factor favouring successful isolation (OR $4.167,95 \%$ CI $1.071-16.216, p=0.046)$. On the contrary, the use of newer enzyme lots was a predisposing factor for poor isolation (OR 0.3846, 95\% CI 0.1426-0.9123, $p=0.043)$.

Initial transplantation outcome Four recipient monkeys were excluded from the islet in vivo functional analysis because they presented severe perioperative complications (bleeding, infection and embolism) and satisfactory follow-up was not possible. The transplants failed to restore euglycaemia and to decrease the insulin requirement to $>50 \%$ of preoperative insulin in three cases $(7 \%)$ because of primary nonfunction. Overall pancreatic islet allotransplantation success for the first two postoperative weeks was 93\%. During this time, 12 monkeys (27\%) were considered to have fully functional allografts, 21 (48\%) had allografts with partial function and eight (18\%) experienced acute rejection. There was a significant correlation between islet yield and graft function (Spearman $r=0.4002, p=0.0002$ ) and between successful isolation and graft function (Spearman $r=0.6769$, $p<0.0001)$. In the univariate analysis, pancreas preservation, enzyme lot and endotoxin level in the digest were the only variables statistically associated with the transplantation outcome (Table 2). In the multivariate analysis, pancreas preservation $(p=0.0447)$, enzyme lot $(p=0.0075)$, endotoxin in the digest $(p<0.0001)$ and COBE collection $(p=0.0166)$ were independent variables contributing to the variability of the transplantation outcome. The chances of having a functional transplant increased by $25 \%$ with the two-layer preservation technique, decreased by $36 \%$ with higher endotoxin levels in the digest, and increased by up to $44 \%$ with a less fractioned COBE collection. 
Table 3 Analysis of successful isolations

\begin{tabular}{|c|c|c|c|}
\hline Variable & Successful isolation $(n=43)$ & Non-successful isolation $(n=29)$ & $p$ value \\
\hline \multicolumn{4}{|l|}{ Criterion } \\
\hline Islet yield (IEQ) & $95829 \pm 38904$ & $47839 \pm 30268$ & $<0.001 *$ \\
\hline Viability $(\%)$ & $95.7 \pm 2.5$ & $88.9 \pm 5.9$ & $<0.001 *$ \\
\hline Purity (\%) & $95.6 \pm 3.4$ & $89.7 \pm 4.9$ & $0.001^{*}$ \\
\hline Endotoxin UW (EU/ml) & $0.28 \pm 0.25$ & $0.64 \pm 0.57$ & $0.022 *$ \\
\hline Endotoxin digest (EU/ml) & $0.56 \pm 0.57$ & $0.86 \pm 0.64$ & $0.026^{*}$ \\
\hline Transplantation & Yes, 36 ; no, $7^{*}$ & Yes, $2 * ;$ no, 27 & $<0.001^{*}$ \\
\hline \multicolumn{4}{|l|}{ Donor variables } \\
\hline Age (years) & $5.2 \pm 1.7$ & $5.9 \pm 2.5$ & 0.457 \\
\hline Sex & Male, 39; female, 4 & Male, 27; female, 2 & 0.638 \\
\hline Donor weight (kg) & $6.8 \pm 2.0$ & $7.5 \pm 2.8$ & 0.368 \\
\hline Medical history & No, 33; yes, 10 & No, 20; yes, 9 & 0.420 \\
\hline Type of monkey & Indian, 29; Chinese, 14 & Indian, 22; Chinese, 7 & 0.613 \\
\hline \multicolumn{4}{|l|}{ Procurement } \\
\hline Surgery findings & Positive, 5 ; negative, 38 & Positive, 23; negative, 6 & 0.885 \\
\hline Pancreas weight & $5.3 \pm 1.7$ & $5.9 \pm 2.5$ & 0.457 \\
\hline Pancreas quality & Adequate, 21 ; inadequate, 22 & Adequate, 15 ; inadequate, 14 & 1.000 \\
\hline Pancreas preservation & UW, 36; two-layer, 7 & UW, 28; two-layer, 1 & 0.126 \\
\hline \multicolumn{4}{|l|}{ Isolation } \\
\hline Volume delivered & $196.7 \pm 129.5$ & $127.3 \pm 98.0$ & $0.039 *$ \\
\hline Ductal infusion & $81.5 \%(0-100)$ & $81.1 \%(1-100)$ & 0.894 \\
\hline Interstitial infusion & $19.4 \%(1-100)$ & $19.4 \%(1-100)$ & 0.984 \\
\hline Infusion method & Pump, 14; hand, 28 & Pump, 3; hand, 25 & $0.046^{*}$ \\
\hline Enzyme lot & $66.9 \%$ & $33.1 \%$ & $<0.001^{*}$ \\
\hline Digestion time (min) & $13.9 \pm 5.1$ & $15.7 \pm 6.1$ & 0.221 \\
\hline Mean temperature $\left({ }^{\circ} \mathrm{C}\right)$ & $33.4 \pm 1.3$ & $33.5 \pm 1.2$ & 0.975 \\
\hline DNAase & Yes, 26; no, 18 & Yes, 17; no, 11 & 1.000 \\
\hline Pefabloc & Yes, $19 ;$ no, 13 & Yes, $10 ;$ no, 13 & 0.285 \\
\hline COBE delivery & Complete, 34 ; fractioned, 9 & Complete, $19 ;$ fractioned, 10 & 0.420 \\
\hline
\end{tabular}

${ }^{*} p<0.05$

\section{Discussion}

Insulin independence after PIT is not yet a consistent achievement in many centres. Several issues in donor standardisation, organ procurement, islet isolation, engraftment and the prevention of allograft rejection still need to be addressed. In order to refine islet transplantation procedures, several methods have been tried in various animal models [17, 18]. Primates are a promising preclinical model for islet transplantation research. Unfortunately there have only been a few reports analysing the results of large-scale NHP islet transplantation $[4,18,19]$. Poor availability of NHP tissue, long learning curves and prohibitive expenses constrain the consistency of isolated NHP islets for PIT studies, which in turn impedes large-scale cooperative studies to improve islet allotransplant results. To advance preclinical studies, we attempted to identify key variables that consistently influence the quantity and quality of NHP islets.

The present work highlights the large number of variables that can influence the outcome of islet isolation and transplant. Although some of the associations found in the univariate analysis may be secondary to confounding variables or statistical errors, the multivariate analysis did distinguish those associations that independently and significantly influenced outcome. The retrospective nature of this study, the long period of time over which it was performed, some missing data and unbalanced frequencies must be considered as possible flaws. Some variables are difficult to control, especially in a long-term retrospective study such as that presented here. Personnel, technology and methods also changed with time. We caution readers to take these factors into consideration when evaluating our observations and to challenge our findings in prospective studies, which will more accurately allow the many variables to be deconvoluted. The best efforts were made to analyse the information using three well-defined outcomes to evaluate islet quantity (islet yield) and quality (successful isolation and transplantation results), allowing us to obtain valuable information to improve PIT. A good islet count does not necessarily mean that the isolation was successful and the islets are ready to be transplanted because a successful isolation combines quantitative and qualitative 
characteristics of the islets. The major parameters favouring successful NHP islet isolation and transplantation were the type of monkey, the two-layer preservation technique, volume of enzyme delivered, enzyme lot, endotoxin level and the type of COBE collection.

The type of monkey was the only donor variable that significantly influenced the islet yield but did not affect the success of isolation and the transplantation outcome. There are many differences in pancreatic isolation results between different mammals, NHPs [4] and humans [20], but we found significant intraspecies differences in favour of the Chinese rhesus macaques as the only significant donor variable. Many variables, such as donor age, BMI, cause of death and the use of vasopressors, considerably influence human PIT $[9,21]$. This individual variability seen in humans is not observed in the experimental setting, where more standardised donors are used, highlighting the importance of donor standardisation in clinical practice $[22,23]$. Therefore, not all the results obtained in NHP PIT may be translated to humans and one should be cautious in generalizing these outcomes. For example, the human pancreas is often fibrotic, and it may therefore be more difficult to infuse large amounts of collagenase solution and ischaemia times (warm and cold) are longer than in the experimental model. Although these differences are important, knowing their performance in different situations and animal models is useful and may have some applications in humans. Our deductions related to successful islet isolation in NHP were derived under relatively ideal conditions, but are probably more relevant to humans than to rodent studies. Translation of these NHP findings to the human cadaveric donor situation is unlikely to be straightforward for the reasons stated above. However, the basic tenets should endure because the process of obtaining islets is similar between species and certain fundamental principles are universal to any islet isolation process.

The two-layer preservation method was another factor favouring higher islet yields and better transplantation results. It has been demonstrated that the two-layer technique oxygenates and activates the metabolism of the pancreas, leading to resuscitation of pancreases with extended preservation time and/or ischaemically damaged tissue [11, 24]. This might explain the higher islet counts and the improved success rate of transplantation compared with the UW preservation method, although the two-layer technique was performed in only eight cases and cold ischaemia times were uniformly short. The small sample size and short ischaemia time could reduce the biological/clinical significance of our observation, but the statistics support the conclusion. Possibly there is a difference in sensitivity of islets taken from healthy normal NHP vs islets from braindead humans with respect to the two-layer preservation method variable. It is not clear in the literature whether the two-layer technique should be used only when the condition of the pancreas is bad or in all procedures [25, 26]. Having analysed these data and the literature, we believe it is reasonable to consider this technique as part of the isolation process, but further studies are required to endorse these observations.

The enzyme lot used in the digestion phase was a major factor influencing islet yield and successful isolation. Enzyme lot number was an independent variable with a significant negative correlation with islet yield (Spearman $r=-0.3707, p=0.0112$ ). Islet yield decreased significantly with newer lots but it was impossible to perform a more detailed investigation because of incomplete data. Despite this observation, the enzyme lot had a strong association with islet yield and isolation success but was more weakly related to transplantation outcome. Assessment of the manufacturer's information on each lot revealed that collagenase content decreased but endotoxin increased in the Liberase during the study period. These data suggest that enzyme quality declined over time, although some other independent factors that were not analysed, such as changes in personnel, the surgeon's skill and protocol modifications, should also be considered. These findings are consistent with recent reports that the efficiency of Liberase HI declines over time in human pancreas processing [27]. We can extrapolate from this that enzyme lots with higher collagenase activity and lower endotoxin content will be advantageous for NHP islet isolation. The duration of pancreas exposure to enzyme demonstrated no association, but the enzyme volume delivered and the method of infusion affected islet yield and isolation success. Complete separation of the exocrine and endocrine pancreas should be a rapid, accurate and uniform procedure. To accomplish this, enzymatic digestion should occur simultaneously from outside and inside the pancreas, to reach every corner of the organ in a homogeneous way [28]. This might be the reason why the volume of enzyme injected into the pancreas before digestion was such a significant factor in the multivariate analysis. The results obtained with the infusion method may be more controversial. Pump infusion demonstrated only a borderline significant association with isolation outcome; this could be due to the comparison of time series and lack of randomisation. Well conducted studies should be performed before arriving at definitive conclusions regarding the superiority of the manual or pump infusion method of introducing enzyme into the pancreas.

Sterility of isolated islets is critical for successful isolation and transplantation. Microbiological assessment and endotoxin levels were used to monitor sterility. Although nonspecific, the endotoxin level was an independent factor associated with islet transplantation success. Notably, endotoxin contamination in UW and Liberase did not influence islet yield per se. This discrepancy between the effects of 
endotoxin levels on islet yield and transplantation success is probably related to the brief exposure of beta cells to endotoxin at low temperature during the isolation process. In contrast, endotoxin contamination in vivo can stimulate production of proinflammatory cytokines that impair beta cell viability and function [15, 16, 29-32]. Endotoxin has been introduced into experimental models by direct contamination or indirectly via commercial products and monitoring and elimination should therefore be performed consistently [33]. Lipopolysaccharide can shift active immunity towards the priming of Th1 cells [34] by promoting activation and maturation of dendritic cells [35], which might be a confounding factor in in vitro and in vivo experimental models. In this way, lipopolysaccharide contamination might antagonise the effects of some immune-modulating agents used in induction protocols (e.g. 5-deoxyspergualin) with potential deleterious effects on tolerance induction. Therefore, lipopolysaccharide monitoring was considered an important part of immune testing in order to avoid misleading results and interpretations. Even though the endotoxin level was not associated with islet yield and isolation success, it was a significant independent factor for transplantation outcome, corroborating the above statements and the observations made by other investigators [15, 16, 29-32].

The method of collection after COBE purification was also an independent factor associated with transplantation success but it did not influence the islet yield and success of isolation. Complete (less fractioned) was superior to multifractioned collection in the multivariate analysis. Most experimental $[4,8,19,36]$ and clinical $[9,22,37]$ studies on pancreatic isolation variables have focused on the type of gradient for islet purification and have found no differences between continuous and discontinuous methods. We used the discontinuous gradient technique, but the sample collecting method was modified during the study period. We hypothesised that collecting more fractions would make it easier to identify where the islets were but the results led us to reject our hypothesis. Since this variable has not been assessed before, it is difficult to find a good explanation for the outcomes. We believe that less manipulation, homogeneous dilutions and fast processing might be the reasons for this difference.

This study provides a guide for donor, isolation, purification and islet characterisation that may improve the efficiency and efficacy of islet transplantation in NHPs. With the caveats of a retrospective study and unbalanced frequencies of some variables, we have identified several key factors that affect the recovery of costly islets isolated from NHPs. However, we recognise that even under the most optimal conditions, islet quantity and quality will be highly variable between isolations. In this retrospective study, the data suggest that future NHP isolations should use bilayer preservation, infuse more than $80 \mathrm{ml}$ of Liberase into the pancreas, collect non-fractioned tissue from the $\mathrm{COBE}$ and strictly monitor for infection.

Acknowledgements This study was supported by NIH Grants 5 U19 DK 57958-07, 1 U19 AI067151-01, U19 AI05642-01 and JDRF grant 4-2004-354 awarded to J. M. Thomas.

Duality of interest J. M. Thomas is a Consultant for Genzyme Corporation. The remaining authors declare that there is no duality of interest associated with this manuscript.

\section{References}

1. Shapiro AM, Lakey JR, Ryan EA et al (2000) Islet transplantation in seven patients with type 1 diabetes mellitus using a glucocorticoidfree immunosuppressive regimen. N Engl J Med 343:230-238

2. Hering BJ, Kandaswamy R, Ansite JD et al (2005) Single-donor, marginal-dose islet transplantation in patients with type 1 diabetes. JAMA 293:830-835

3. Scharp DW, Murphy JJ, Newton WT, Ballinger WF, Lacy PE (1975) Transplantation of islets of Langerhans in diabetic rhesus monkeys. Surgery 77:100-105

4. Ranuncoli A, Cautero N, Ricordi C et al (2000) Islet cell transplantation: in vivo and in vitro functional assessment of nonhuman primate pancreatic islets. Cell Transplant 9:409-414

5. Gray DW (1990) Islet isolation and transplantation techniques in the primate. Surg Gynecol Obstet 170:225-232

6. O'Neil JJ, Tchipashvili V, Parent RJ et al (2003) A simple and cost-effective method for the isolation of islets from nonhuman primates. Cell Transplant 12:883-890

7. Thomas JM, Contreras JL, Smyth CA et al (2001) Successful reversal of streptozotocin-induced diabetes with stable allogeneic islet function in a preclinical model of type 1 diabetes. Diabetes 50:1227-1236

8. Matsumoto S, Iwanaga Y, Okitsu T et al (2005) Analysis of largescale nonhuman primate islet isolations. Transplant Proc 37:13171321

9. Nano R, Clissi B, Melzi R et al (2005) Islet isolation for allotransplantation: variables associated with successful islet yield and graft function. Diabetologia 48:906-912

10. Shibata S, Kirchhof N, Matsumoto S et al (2002) High-dose streptozotocin for diabetes induction in adult rhesus monkeys. Transplant Proc 34:1341-1344

11. Matsumoto S, Qualley SA, Goel S et al (2002) Effect of the twolayer (University of Wisconsin solution-perfluorochemical plus $\mathrm{O}_{2}$ ) method of pancreas preservation on human islet isolation, as assessed by the Edmonton Isolation Protocol. Transplantation 74:1414-1419

12. Ricordi C, Lacy PE, Finke EH, Olack BJ, Scharp DW (1988) Automated method for isolation of human pancreatic islets. Diabetes 37:413-420

13. Kenyon NS, Chatzipetrou M, Masetti M et al (1999) Long-term survival and function of intrahepatic islet allografts in rhesus monkeys treated with humanized anti-CD154. Proc Natl Acad Sci USA 96:8132-8137

14. Ricordi C, Gray DW, Hering BJ et al (1990) Islet isolation assessment in man and large animals. Acta Diabetol Lat 27:185-195

15. Berney T, Molano RD, Cattan P et al (2001) Endotoxin-mediated delayed islet graft function is associated with increased intra-islet cytokine production and islet cell apoptosis. Transplantation $71: 125-132$

16. Linetsky E, Inverardi L, Kenyon NS, Alejandro R, Ricordi C (1998) Endotoxin contamination of reagents used during isolation 
and purification of human pancreatic islets. Transplant Proc 30:345-346

17. Lakey JR, Cavanagh TJ, Zieger MA, Wright M (1998) Evaluation of a purified enzyme blend for the recovery and function of canine pancreatic islets. Cell Transplant 7:365-372

18. Dufrane D, D'Hoore W, Goebbels RM, Saliez A, Guiot Y, Gianello P (2006) Parameters favouring successful adult pig islet isolations for xenotransplantation in pig-to-primate models. Xenotransplantation 13:204-214

19. Balamurugan AN, Ramakrishna B, Gunasekaran S (2004) Insulin secretory characteristics of monkey pancreatic islets: a simple method of islet isolation and the effect of various density gradients on separation. Diabetes Res Clin Pract 66:13-21

20. Brandhorst D, Brandhorst H, Hering BJ, Federlin K, Bretzel RG (1995) Islet isolation from the pancreas of large mammals and humans: 10 years of experience. Exp Clin Endocrinol Diabetes 103( Suppl 2):3-14

21. Lakey JR, Warnock GL, Rajotte RV et al (1996) Variables in organ donors that affect the recovery of human islets of Langerhans. Transplantation 61:1047-1053

22. Matsumoto S, Zhang G, Qualley S et al (2004) Analysis of donor factors affecting human islet isolation with current isolation protocol. Transplant Proc 36:1034-1036

23. O'Gorman D, Kin T, Murdoch T et al (2005) The standardization of pancreatic donors for islet isolations. Transplantation 80:801-806

24. Matsumoto S, Zhang G, Qualley S et al (2004) The effect of twolayer (University of Wisconsin solution/perfluorochemical) preservation method on clinical grade pancreata prior to islet isolation and transplantation. Transplant Proc 36:1037-1039

25. Fraker CA, Alejandro R, Ricordi C (2002) Use of oxygenated perfluorocarbon toward making every pancreas count. Transplantation 74:1811-1812

26. Lakey JR, Tsujimura T, Shapiro AM, Kuroda Y (2002) Preservation of the human pancreas before islet isolation using a two-layer (UW solution-perfluorochemical) cold storage method. Transplantation 74:1809-1811
27. Yamamoto T, Ricordi C, Messinger S et al (2007) Deterioration and variability of highly purified collagenase blends used in clinical islet isolation. Transplantation 84:997-1002

28. Andrades P, Asiedu C, Ray P et al (2007) Islet yield after different methods of pancreatic Liberase delivery. Transplant Proc 39: $183-184$

29. Jahr H, Pfeiffer G, Hering BJ, Federlin K, Bretzel RG (1999) Endotoxin-mediated activation of cytokine production in human PBMCs by collagenase and Ficoll. J Mol Med 77:118-120

30. Linetsky E, Bottino R, Lehmann R, Alejandro R, Inverardi L, Ricordi C (1997) Improved human islet isolation using a new enzyme blend, liberase. Diabetes 46:1120-1123

31. Vargas F, Vives-Pi M, Somoza N et al (1998) Endotoxin contamination may be responsible for the unexplained failure of human pancreatic islet transplantation. Transplantation 65: $722-727$

32. Vives-Pi M, Somoza N, Fernandez-Alvarez J et al (2003) Evidence of expression of endotoxin receptors CD14, toll-like receptors TLR4 and TLR2 and associated molecule MD-2 and of sensitivity to endotoxin (LPS) in islet beta cells. Clin Exp Immunol 133:208-218

33. Petsch D, Anspach FB (2000) Endotoxin removal from protein solutions. J Biotechnol 76:97-119

34. Zimmer S, Pollard V, Marshall GD et al (1996) The 1996 Moyer Award. Effects of endotoxin on the Th1/Th2 response in humans. J Burn Care Rehabil 17:491-496

35. Saito Y, Yanagawa Y, Kikuchi K, Iijima N, Iwabuchi K, Onoe K (2006) Low-dose lipopolysaccharide modifies the production of IL-12 by dendritic cells in response to various cytokines. J Clin Exp Hematop 46:31-36

36. Selvaggi G, Fernandez L, Bottino R et al (1997) Improved baboon pancreatic islet cell isolation. Transplant Proc 29:1967-1968

37. Kim SC, Han DJ, Kang CH et al (2005) Analysis on donor and isolation-related factors of successful isolation of human islet of Langerhans from human cadaveric donors. Transplant Proc 37:3402-3403 